\title{
Chapter 8. Hypertension in the elderly
}

\author{
Hypertension Research (2009) 32, 57-62; doi:10.1038/hr.2008.6
}

\section{POINT 8}

1. Treatment sufficient to achieve the final target of blood pressure control should be performed in elderly patients. Prognosis is expected to improve by reducing blood pressure to $<140 / 90 \mathrm{~mm} \mathrm{Hg}$ at all ages. In patients that have been well treated before the age of 65 years, the same antihypertensive treatment should be continued after the age of 65 years.

2. Blood pressure should be reduced gradually, with due attention to the appearance of adverse effects.

3. Nondrug therapies should be conducted by determining approaches individually, with regard to the quality of life (QOL).

4. In antihypertensive drug treatment, a calcium (Ca) antagonist, angiotensin receptor blocker (ARB), agiotensin-converting enzyme (ACE) inhibitor or a low-dose diuretic should be the first choice. Generally, treatment should be started at half the routine dose. If the antihypertensive effect is insufficient, these drugs should be used in combination.

5. If there are complications, the optimal antihypertensive drug should be selected for each patient by referring to Chapter 6 and Chapter 7. Blood pressure should be reduced carefully by paying attention to organ damage and QOL.

\section{1) CHARACTERISTICS OF HYPERTENSION IN THE ELDERLY}

Japan is a super-aged society, in which the elderly account for approximately $21 \%$ of the population and those aged $\geqslant 75$ years account for approximately $10 \%$. Hypertension increases with age, and, according to the National Health and Nutrition Survey of Japan (2006), $61 \%$ of those in their $60 \mathrm{~s}$ and $72 \%$ of those aged above 70 years have hypertension.

Systolic pressure increases, but diastolic pressure tends to decrease, with age, causing an increase in pulse pressure. These increases in systolic and pulse pressure are important risk factors for cardiovascular disease in elderly people. The rise in pulse pressure is the result of a decline in the Windkessel function owing to a decrease in the extensibility of the aortic wall associated with the progression of atherosclerosis. According to the Hisayama Study, in which participants were aged $\geqslant 60$ years; the cumulative incidence of cardiovascular disease increased significantly when systolic pressure was $\geqslant 140 \mathrm{~mm} \mathrm{Hg}$ and diastolic pressure was $\geqslant 80 \mathrm{~mm} \mathrm{Hg} .{ }^{8}$

The hemodynamics of hypertension in the elderly is characterized by arteriosclerosis and a decrease in vascular elasticity, a decrease in the baroreceptor reflex, cardiac hypertrophy and a decrease in the diastolic function of the left ventricle, and impairment of body fluid volume regulation, with a consequent decrease in blood flow and dysfunction of autoregulation in major organs. In addition, autoregulation of the blood flow of target organs is impaired, and the lower limit of blood pressure shifts toward the hypertensive side. For this reason, a rapid and marked decrease in blood pressure may cause circulatory disorders of such organs and hence blood pressure must be reduced with care. Similar attention must be paid to renal dysfunction and adverse effects due to an excessive decrease in blood pressure. However, long-term control of blood pressure is clearly effective in preventing cardiovascular events and progression of organ damage. Therefore, aggressive control of the blood pressure is also desirable in elderly patients.

Among metabolic characteristics, increases in susceptibility to disturbances of electrolyte homeostasis (particularly, hyponatremia and hypokalemia), insulin resistance and glucose intolerance are important in elderly patients.

Characteristics of blood pressure in elderly patients with hypertension include (1) increasing systolic and pulse pressure, (2) instability of blood pressure, (3) increase in orthostatic and postprandial hypotension, (4) increase in the nondipper-type nighttime blood pressure, (5) increase in morning surge, (6) increase in white coat hypertension, (7) presence of patients with auscultatory gaps (disappearance of Korotkoff sounds) and (8) presence of pseudohypertension (a higher blood pressure indicated by the manchette method compared with the direct method; however, the frequency of pseudohypertension is low in evaluations in Japan). Impairment of both the pressor and depressor systems, including the nervous system (impairment of baroreceptor reflex, impairment of $\beta$-receptor function and so on) and humoral blood pressure-regulating mechanisms (decline in the renin-angiotensin system, kallikrein-kinin system, prostaglandin system, renal dopamine system and so on), are involved in these phenomena.

\section{2) CRITERION FOR HYPERTENSION IN THE ELDERLY AND RESULTS OF EPIDEMIOLOGICAL STUDIES}

The criterion for hypertension should be blood pressure $\geqslant 140 /$ $90 \mathrm{~mm} \mathrm{Hg}$ for elderly people as well as for adults in general. A positive correlation was observed between increase in blood pressure and elevation in cardiovascular risk in the Hisayama Study ${ }^{8}$ and in the meta-analysis ${ }^{9}$ of approximately one million people with no history of cardiovascular disease in 61 prospective studies of people in old age. This correlation was still observed in those in their 80s, and absolute cardiovascular risk increased with age whereas the slope of correlation became gentler in old age. In NIPPON DATA80, which is a 19-year follow-up study in Japan, an increase in blood pressure was also positively correlated with an increase in the risk of cardiovascular death. ${ }^{20}$ On the basis of these results, the same 
criterion of hypertension as that for adults in general was set for elderly people.

However, there are epidemiological studies that report the presence of a blood pressure threshold related to increases in cardiovascular risk and mortality rate. On re-analysis of the results of the Framingham Study, ${ }^{541}$ in which the threshold of blood pressure for an increase in mortality was calculated, blood pressure was found to increase with age and was approximately $160 \mathrm{~mm} \mathrm{Hg}$ for men and approximately $170 \mathrm{~mm} \mathrm{Hg}$ for women in early old age (65-74 years). In the Hisayama Study, the risk increased significantly to $\geqslant 140 \mathrm{~mm} \mathrm{Hg}$ in those aged between 60 and 79 years and $\geqslant 180 \mathrm{~mm} \mathrm{Hg}$ in those aged $\geqslant 80$ years. ${ }^{8}$

Furthermore, in prospective studies in local residents aged $\geqslant 85$ years, a negative correlation was noted between blood pressure and life span. In Vantaa $85+{ }^{542}$ and Leiden $85+,{ }^{543}$ performed in separate populations, the outcome was better in the group with systolic pressure of $\geqslant 160 \mathrm{~mm} \mathrm{Hg}$ than in the group with systolic pressure $<140 \mathrm{~mm} \mathrm{Hg}$.

Taken together, it is generally considered that cardiovascular risk is lower at a lower blood pressure even in elderly people. The discrepancies in the results depending on the study participants and analysis outcomes suggest that the clinical importance of hypertension differs among subpopulations of elderly people classified by age and pathological condition. However, these are the results of epidemiological studies and do not indicate criteria useful for intervention with antihypertensive drug therapy.

\section{3) DIAGNOSIS}

\section{a. Diagnosis considering fluctuation of blood pressure}

As blood pressure fluctuates markedly in elderly patients with hypertension, it must be measured repeatedly on different days to confirm that it is consistently high. As the frequency of orthostatic hypotension increases, the measurement of blood pressure in the standing position (within 3 min after standing up) is important and must be performed before and after the beginning of treatment. Blood pressure should be measured simultaneously by the palpation method to avoid overlooking pseudohypertension and auscultatory gaps. Home blood pressure measurement and 24-h ambulatory blood pressure monitoring are both useful for evaluating the fluctuation of blood pressure, white coat hypertension, morning hypertension and masked hypertension (reverse white coat hypertension) in daily practice. Masked hypertension has been reported to increase cardiovascular risk in elderly participants (mean age, 70 years). ${ }^{88}$

\section{b. Differential diagnosis of secondary hypertension}

Attention to secondary hypertension should be paid, particularly to renovascular hypertension due to atherosclerosis and primary aldosteronism, an endocrine hypertension. Secondary hypertension is indicated in patients who show a significant increase in blood pressure within a short term, an exacerbation of blood pressure control and refractory hypertension. Auscultation of abdominal vascular murmur is useful for the diagnosis of renovascular hypertension. If a rapid decline in renal function is observed during treatment with an ACE inhibitor or ARB, bilateral renovascular hypertension should be suspected. If a tendency toward hypokalemia is noted, primary aldosteronism should be suspected. Attention to drug-induced hypertension is also necessary.

\section{c. Diagnosis of target organ damage and complications}

The presence or absence of damage to target organs such as the brain, heart and kidneys owing to hypertension is important for the determination of therapeutic approach and selection of drugs in elderly patients as well as in adults in general. As elderly patients often show asymptomatic multiple organ damage, efforts to detect latent complications are necessary. Reports suggest that attention to a decrease in diastolic pressure is also necessary in patients with systolic hypertension complicated by ischemic heart disease, ${ }^{544}$ and that reducing blood pressure increases the risk of stroke in patients with $75 \%$ or more stenosis of the bilateral carotid arteries, ${ }^{324}$ so that particular attention is required in such patients. Regarding the relationship between the diagnosis of complications and treatment, the presence or absence of complications such as respiratory diseases (particularly obstructive pulmonary disease) and metabolic complications (diabetes mellitus, dyslipidemia and hypokalemia) is also important for the choice of antihypertensive drugs.

\section{4) TREATMENT}

\section{a. Effects of antihypertensive therapy in the elderly}

Randomized controlled studies using a placebo as a control have shown the preventive effects of antihypertensive drugs against cardiovascular diseases in elderly hypertensive participants aged $\geqslant 60$ or 70 years ${ }^{54}$-in EWPHE, STOP-Hypertension and MRC II using diuretics and $\beta$-blockers, and in the SCOPE using ARBs. As for isolated systolic hypertension, which is commonly observed in elderly people, it is shown by SHEP using diuretics and by Syst-Eur, Syst-China and STONE using calcium (Ca) antagonists. Hypertension with a systolic pressure of $\geqslant 160 \mathrm{~mm} \mathrm{Hg}$ was treated in many of these studies, and mean systolic pressure was reduced from 166-197 $\mathrm{mm} \mathrm{Hg}$ before treatment to almost $140-150 \mathrm{~mm} \mathrm{Hg}$.

According to a meta-analysis of nine major clinical studies on the treatment of hypertension in elderly patients, antihypertensive drug treatment significantly reduced all-cause death by $12 \%$, death from stroke by $36 \%$ and death from ischemic heart disease by $25 \%$; it also significantly reduced the incidence of cerebrovascular disease by $35 \%$ and the incidence of ischemic heart disease by $15 \%{ }^{546}$ In HYVET, involving patients with hypertension (mean blood pressure: $173 / 91 \mathrm{~mm} \mathrm{Hg}$ ) aged $\geqslant 80$ years, treatment was performed using diuretics (ACE inhibitors were added when the antihypertensive effect was insufficient) with a target of $<150$ / $80 \mathrm{~mm} \mathrm{Hg}$, and a significant $30 \%$ decrease in death from stroke, $21 \%$ decrease in death from any cause, $64 \%$ decrease in heart failure and $34 \%$ decrease in cardiovascular events were observed. ${ }^{182}$ In the second year, blood pressure was reduced to $144 / 78 \mathrm{~mm} \mathrm{Hg}$ in the active treatment group.

Thus, adequate antihypertensive treatment is also recommended in elderly patients. However, HYVET or other studies do not provide clear evidence supporting the usefulness of reducing blood pressure to $<140 \mathrm{~mm} \mathrm{Hg}$ in those patients aged $\geqslant 80$ years or antihypertensive treatment for grade I hypertension in elderly people; therefore, blood pressure must be reduced cautiously.

\section{b. Indications for antihypertensive therapy and target levels of blood pressure}

Indications for treatment. Indications for antihypertensive treatment are evaluated from patients' characteristics obtained from randomized placebo-controlled studies in which the usefulness of antihypertensive treatment was confirmed. In studies involving only elderly patients with hypertension, those with a systolic pressure of $\geqslant 160 \mathrm{~mm} \mathrm{Hg}$ and a diastolic pressure of $\geqslant 90-100 \mathrm{~mm} \mathrm{Hg}$ were selected as participants. In contrast, there is no clear evidence supporting the usefulness of antihypertensive treatment in elderly patients with grade I hypertension at a moderate risk level, but it has been reported that a $\mathrm{Ca}$ antagonist regressed cardiac hypertrophy and improved the 
quality of life (QOL) ${ }^{547}$ From these observations, elderly patients with a blood pressure of $\geqslant 140 / 90 \mathrm{~mm} \mathrm{Hg}$ are regarded as indicators for treatment.

Target control levels. In elderly patients, sufficient antihypertensive treatment should be performed with a target of $<140 / 90 \mathrm{~mm} \mathrm{Hg}$, which is a criterion for hypertension. In patients who have been treated before the age of 65 years and have controlled blood pressure at $<130 / 85 \mathrm{~mm} \mathrm{Hg}$, there is no need to attenuate treatment after the age of 65 years.

Blood pressure levels obtained in large clinical studies and the results of comparisons among the groups that were set at different target levels provide useful information for setting the target blood pressure. In recent large clinical studies in elderly patients with hypertension, the mean systolic and diastolic pressures after treatment were $141-152$ and $77-85 \mathrm{~mm} \mathrm{Hg}$, respectively (Table 8-1). In many studies using antihypertensive drugs in which about half of the participants were elderly, ${ }^{198,295,319}$ blood pressure could also be reduced to $<140 / 90 \mathrm{~mm} \mathrm{Hg}$, and the usefulness of antihypertensive treatment was confirmed, although some of these studies included participants without hypertension.

The strategy that reducing blood pressure to $<140 \mathrm{~mm} \mathrm{Hg}$ in elderly patients with a systolic pressure of $\geqslant 160 \mathrm{~mm} \mathrm{Hg}$ was not borne out by the results of SHEP, a subanalysis of HOT and JATOS. ${ }^{548}$ In SHEP, treatment most notably reduced the risk of stroke in the group in which blood pressure was reduced to $<150 \mathrm{~mm} \mathrm{Hg}$, but the significance of this effect disappeared in the group in which blood pressure was reduced to $<140 \mathrm{~mm} \mathrm{Hg}{ }^{549}$ In HOT, which was a group comparative study regarding the target of blood pressure control, thelower-the-better relationship disappeared in participants aged $\geqslant 65$ years. ${ }^{550}$ According to the results of JATOS, which performed a group comparison related to target control level in elderly patients with hypertension, no difference was noted in the outcome, including those with cardiovascular disease, between the group treated with a target of $<140 \mathrm{~mm} \mathrm{Hg}$ and that treated with a target of $140-160 \mathrm{~mm} \mathrm{Hg}$. Whether the outcome is improved by strict control of blood pressure in elderly people is presently unclear from the results of group comparative studies on target control level.

As for the risk of an excessive decrease in blood pressure, analysis of diastolic blood pressure achieved has provided retrospective evidence supporting the J-shaped phenomenon, but the threshold has not been consistent, and no clear conclusion has been reached with regard to the presence of the J-shaped phenomenon for systolic pressure. In SHEP, cardiovascular events increased when diastolic pressure was $<60 \mathrm{~mm} \mathrm{Hg}$. ${ }^{51}$ Subanalysis of Syst-Eur warned against reducing diastolic blood pressure to $<70 \mathrm{~mm} \mathrm{Hg}$ in patients with systolic hypertension complicated by ischemic heart disease. ${ }^{544}$ In PATEHypertension, ${ }^{552}$ performed in Japan, cardiovascular events increased when systolic pressure was reduced to $<120 \mathrm{~mm} \mathrm{Hg}$, but the total number of events was small, and further evaluation is necessary. In recent large clinical studies that enrolled a large number of elderly patients with hypertension, such as INSIGHT, ALLHAT and VALUE, the J-shaped phenomenon was not observed even when blood pressure was reduced to approximately $135-138 / 75-82 \mathrm{~mm} \mathrm{Hg}$. In JATOS $^{548}$ and a subanalysis of CASE-J, ${ }^{298}$ in addition, a target of $<140 \mathrm{~mm} \mathrm{Hg}$ was achieved in elderly patients without an increase in adverse events. Therefore, although the risk of an excessive decrease in blood pressure is generally low, the target level of systolic pressure should be attained with sufficient attention to changes in the diastolic pressure and ischemic heart disease in patients with low diastolic pressure.
Gradual reduction in blood pressure. In elderly patients with hypertension, particular attention must be paid to the speed of blood pressure reduction because of organ circulation disorders and impairment of autoregulation. Blood pressure should be reduced gradually, antihypertensive drug treatment should be started generally at half the regular dose, and the dose should be increased at an interval of 4 weeks to 3 months by evaluating the presence or absence of signs of brain ischemia, such as dizziness and orthostatic dizziness, symptoms of angina pectoris, ECG changes indicating myocardial ischemia and decline in the QOL. Although $<140 / 90 \mathrm{~mm} \mathrm{Hg}$ should be the final target of blood pressure control in patients aged $\geqslant 75$ years with grade II or III hypertension $(\geqslant 160 \mathrm{~mm} \mathrm{Hg})$, blood pressure should be reduced carefully by setting an intermediate target of $<150 / 90 \mathrm{mmHg}$. This recommendation is supported by the results of JATOS ${ }^{548}$ and the subanalysis of CASE- ${ }^{298}$ performed in Japan. In HYVET, in which the participants were patients aged $\geqslant 80$ years with grade II or more severe hypertension, target control level was $<150 / 80 \mathrm{~mm} \mathrm{Hg}$, and whether the dose should be increased was evaluated every 3 months and the mean blood pressure in the treated group after 2 years was $144 / 78 \mathrm{~mm} \mathrm{Hg}{ }^{182}$ Blood pressure should also be reduced slowly and carefully in patients with a large pulse pressure, because advanced atherosclerosis is suspected.

\section{c. Lifestyle modifications}

In elderly people, nonpharmacological therapies (lifestyle modifications), such as restriction of salt intake and exercise and weight control, are beneficial and should be practiced at all cost. However, marked changes in lifestyle may impair QOL, and hence lifestyle modifications should be limited to a stress-free level.

Dietary therapy. As elderly people generally have high salt sensitivity, salt intake restriction is effective. The target of salt intake restriction should be $6 \mathrm{~g} \mathrm{day}^{-1}$, but caution is needed, because excessive salt intake restriction may cause dehydration. Weight control is also effective in obese individuals. ${ }^{190}$ Potassium intake has a mild antihypertensive effect and contributes to the prevention of cardiovascular disease such as stroke. Generally, a potassium-rich diet is recommended, but attention to hyperkalemia associated with renal dysfunction or diabetes is necessary, and potassium intake should be restricted in such patients.

Calcium and magnesium $(\mathrm{Mg})$ intakes have been reported to be negatively correlated with blood pressure, and increasing Ca intake $\left(\geqslant 800 \mathrm{mg} \mathrm{day}^{-1}\right)$ should also be encouraged for the prevention of osteoporosis. Mg supplementation has been shown to have a mild antihypertensive effect.

Exercise therapy. Grade I hypertension was also a good indication for exercise therapy in elderly patients with a mean age of 75 years. ${ }^{553}$ For those aged $\geqslant 60$ years, mild exercise at a heart rate of approximately 110 b.p.m. (beats per minute; fast walking and so on) should be performed regularly for $30-40 \mathrm{~min}$ per session, and three or more times a week. However, exercise is impossible if there are complications such as ischemic heart disease, heart failure, renal insufficiency and joint disease.

Drinking and smoking. The level of alcohol intake is positively correlated with blood pressure and alcohol intake should be restricted to $\leqslant 20-30 \mathrm{ml}$ ethanol per day in elderly people. Although the effect of smoking on blood pressure is mild, it is a strong risk factor for cardiovascular disease and therefore the patient should quit smoking, in principle. 
Table 8-1 Major clinical studies in elderly patients with hypertension and those performed in Japan

\begin{tabular}{|c|c|c|c|c|c|c|c|c|}
\hline & STOP-2 & $A N B P-2$ & SCOPE & HYVET & NICS-EH & PATE-HT & JATOS & $\begin{array}{l}\text { CASE-J } \\
\text { (subanalysis) }\end{array}$ \\
\hline $\begin{array}{l}\text { Participants' } \\
\text { age (years) }\end{array}$ & $70-84$ & $65-84$ & $70-89$ & $\geqslant 80$ & $\geqslant 60$ & $\geqslant 60$ & $65-85$ & $75-84$ \\
\hline Mean age (years) & 76 & 71.9 & 76.4 & 83.6 & 69.8 & 70 & 73.6 & 78.3 \\
\hline $\begin{array}{l}\text { Number of } \\
\text { participants }\end{array}$ & 6614 & 6083 & 4964 & 3845 & 414 & 1748 & 4418 & 751 \\
\hline Study design & PROBE & PROBE & Double-blind & Double-blind & Double-blind & Open & PROBE & PROBE \\
\hline $\begin{array}{l}\text { Follow-up period } \\
\text { (years) }\end{array}$ & 4 & 4.1 & 3.7 & 2.1 & 5 & 2.4 & 2 & 3.2 \\
\hline $\begin{array}{l}\text { after treatment } \\
(\mathrm{mm} \mathrm{Hg})\end{array}$ & (2) $159 / 80$ & (2)142/79 & (2) $149 / 82$ & (2)159/84 & (2) $147 / 79$ & (2)141/78 & (2)146/78 & \\
\hline Primary end point & CV death & CV event & CV event & Stroke & CV event & CV event & CV event & CV event \\
\hline $\begin{array}{l}\text { Results of primary } \\
\text { end point }\end{array}$ & NS & $\begin{array}{l}\text { (1) was useful } \\
(P=0.05)\end{array}$ & NS & $\begin{array}{l}\text { (1) was useful } \\
(P=0.055)\end{array}$ & NS & NS & NS & NS \\
\hline Other results & & $\begin{array}{l}\text { (1) was useful } \\
\text { in men }\end{array}$ & $\begin{array}{l}\text { (1) reduced } \\
\text { lethal stroke } \\
(P=0.04)\end{array}$ & $\begin{array}{l}(1) \text { reduced } \\
\text { total mortality } \\
\text { by } 21 \% \\
(P=0.019)\end{array}$ & & $\begin{array}{l}\text { Cardiac events } \\
\text { increased when } \\
\text { the blood pressure } \\
\text { was reduced to } \\
<120 \mathrm{~mm} \mathrm{Hg}\end{array}$ & & $\begin{array}{l}\mathrm{CV} \text { events increased } \\
\text { when the blood } \\
\text { pressure remained } \\
\text { at } \geqslant 150 \mathrm{~mm} \mathrm{Hg}\end{array}$ \\
\hline
\end{tabular}

Abbreviations: $\mathrm{CV}$, cardiovascular; NS, not significant.

Blood pressure values after treatment in the HYVET comprised data in the second year. The results for the primary evaluation item in the HYVET were not significant because the study was discontinued because of a significant decrease in total mortality. Therefore, the table shows that (1) was useful.

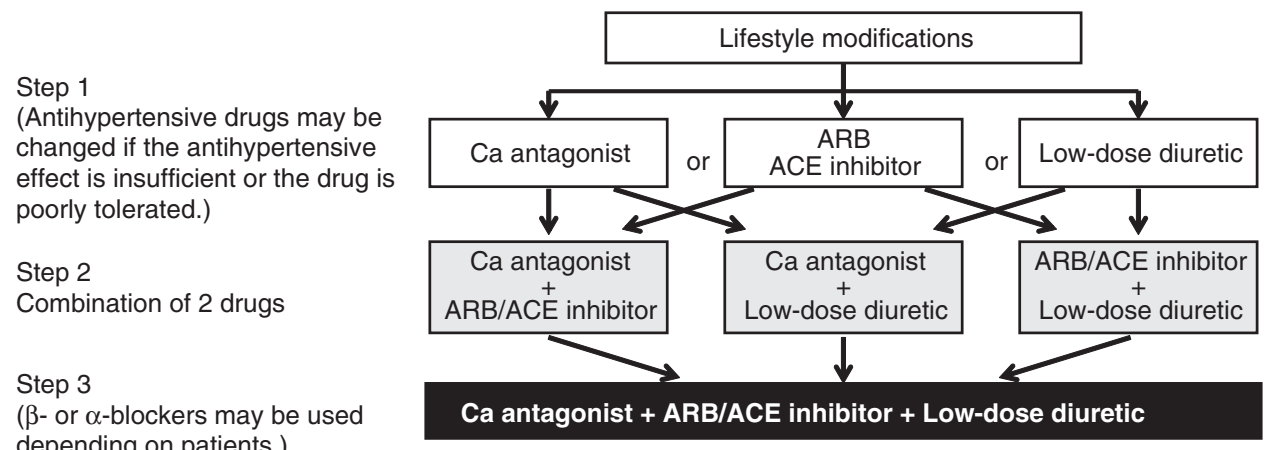

Figure 8-1 Treatment algorithm for elderly patients with hypertension. Antihypertensive drug treatment should be started generally at half the regular dose, and the dose should be increased at an interval of 4 weeks to 3 months. The final target of blood pressure is $<140 / 90 \mathrm{mmHg}$. In patients aged $\geqslant 75$ years with grade II or III hypertension ( $\geqslant 160 \mathrm{mmHg}$ ), blood pressure should be reduced carefully by setting an intermediate target of $<150 / 90 \mathrm{mmHg}$.

\section{d. Choice of antihypertensive drugs}

Antihypertensive drugs must be selected considering the characteristics of hypertension in elderly patients, such as reduced organ blood flow, impairment of autoregulation and orthostatic hypotension, and according to complications if they are present. In the following sections, the evidence-based selection of drugs is discussed by assuming that positive indications are present when the patients have specific complications and absent otherwise.
Patients without complications. A Ca antagonist, ARB, ACE inhibitor or a low-dose thiazide diuretic is appropriate as the first choice. Figure 8-1 shows the serial steps of treatment. If the antihypertensive effect is insufficient, or if the treatment is poorly tolerated, the first choice may be replaced by another drug. If the antihypertensive effect is insufficient with a single drug, combination therapy should be conducted according to Figure 8-1.

The effectiveness of $\mathrm{Ca}$ antagonists for the treatment of hypertension in the elderly, including isolated systolic hypertension, has been 
established by studies ${ }^{545}$ such as STONE, Syst-Eur, Syst-China, NICS$\mathrm{EH},{ }^{276}$ STOP-Hypertension-2, ${ }^{259}$ PATE-Hypertension, ${ }^{552}$ and CASE-J subanalysis. ${ }^{298}$ Subanalysis of the Syst-Eur also suggested that a Ca antagonist (nitrendipine) has a preventive effect on dementia (particularly Alzheimer's disease) ${ }^{555}$ It has few contraindications and can be used with a wide range of other antihypertensive drugs. Attention to conduction disorder, bradycardia and heart failure is necessary on using diltiazem. Ca antagonists are the antihypertensive drugs used most widely in Japan today as many meta-analyses have indicated that they have an excellent stroke-preventing effect, ${ }^{255}$ and hence they are highly useful in Japan, where stroke is a major complication of hypertension.

ARBs can also be the first choice as they have few adverse effects, excellent tolerability and produces a sufficient antihypertensive effect in elderly hypertensive patients. The LIFE ${ }^{556}$ and SCOPE, ${ }^{557}$ in which subanalyses regarding isolated systolic hypertension, a condition frequently observed in elderly people, were performed, are considered to have established the usefulness of ARBs in elderly patients with hypertension. Furthermore, in the subanalysis of the CASE-J involving elderly people, ${ }^{298}$ the group treated with an ARB (candesartan) showed no difference in the incidence of events from the group treated with a $\mathrm{Ca}$ antagonist (amlodipine), the usefulness of which has been established in elderly patients with hypertension. The percentage of candesartan-treated patients who received other antihypertensive drugs was larger than that of the amlodipine-treated patients (54.5 and 42.7\%) in CASE-J. ${ }^{195}$ Therefore, combination therapy should be positively considered to achieve the target blood pressure strictly in elderly patients.

In the STOP-Hypertension-2, the effectiveness of ACE inhibitors for the prevention of cardiovascular events in elderly patients with hypertension was comparable to that of conventional antihypertensive drugs such as diuretics and that of Ca antagonists. ${ }^{259}$ In the ANBP2, an ACE inhibitor (enalapril) was more effective than diuretics, particularly in men. ${ }^{558}$ According to the PATE-Hypertension performed in Japan, in which elderly patients with hypertension (aged $\geqslant 60$ years) under treatment were followed up for 3 years, no significant difference was observed in the incidence of cardiovascular complications between the $\mathrm{Ca}$ antagonist (manidipine) and ACE inhibitor (delapril) groups, although it was not a randomized controlled study. However, the dropout rate was significantly higher in the ACE inhibitor group than in the $\mathrm{Ca}$ antagonist group, and many dropouts were because due to cough. ${ }^{552}$ In elderly patients, aspiration pneumonia accounts for a high percentage of pneumonia and is often related to survival. ACE inhibitors have been reported to reduce the frequency of aspiration pneumonia by enhancing the cough reflex. ${ }^{532,533}$

The usefulness of diuretics has been established by many large-scale clinical studies, including the EWPHE, SHEP and STOP-Hypertension. In the HYVET, in which the participants were patients with grade II or severer hypertension aged $\geqslant 80$ years, a diuretic (indapamide) was used as the basic drug in the antihypertensive treatment group. ${ }^{182}$ An ACE inhibitor (perindopril) was used as an additional antihypertensive drug to achieve the target blood pressure $(<150 /$ $80 \mathrm{~mm} \mathrm{Hg}$ ), and these drugs were used in combination in $73 \%$ of the patients in the second year of the study. The NICS-EH, a double-blind randomized controlled study in elderly patients with hypertension (aged $\geqslant 60$ years, with a mean age of $69.8 \pm 6.5$ years) performed in Japan, showed no difference in the incidence of cardiovascular complications between the Ca antagonist (sustained-release nicardipine) group and the diuretic (trichlormethiazide) group. The tolerability based on the medical dropout rate tended to be higher in the Ca antagonist group. ${ }^{276}$ In the ANBP2, the risk of lethal stroke was significantly lower in the diuretic group than in the ACE inhibitor group. ${ }^{558}$ Diuretics should be used at a low dose with due attention to the effects on complications such as impairment of glucose tolerance, hyperuricemia and dyslipidemia. Diuretics are extremely useful as drugs in combination with $\mathrm{Ca}$ antagonists, ARB and ACE inhibitors.

As for $\beta$-blockers, which are a first choice for adults in general, some meta-analyses failed to establish their preventive effect on ischemic heart disease, cardiovascular death or total death in elderly patients with hypertension, ${ }^{277}$ but others indicated that they are equally effective for reducing the cardiovascular risk compared with the drugs shown in Figure 8-1. ${ }^{559}$ However, elderly patients often have contraindications to $\beta$-blockers or conditions that require caution in their use, so that a $\beta$-blocker is not recommended as the first choice for hypertension in elderly patients.

$\alpha$-Blockers are effective in treating patients with prostatic hypertrophy, but they are not recommended as the first choice because of the high prevalence of orthostatic hypotension in elderly people.

Patients with complications. As elderly patients with hypertension often have complications, the target blood pressure and choice of antihypertensive drugs should be considered individually depending on the complications. Complication of hypertension by cerebrovascular diseases, nephropathy, ischemic heart disease, diabetes mellitus and dyslipidemia creates a high-risk state that generally requires more aggressive antihypertensive treatment. Table 8-2 shows whether various antihypertensive drugs are indicated or contraindicated for various complications. The Table includes conditions frequently observed in, or characteristic of, elderly people, such as chronic obstructive pulmonary disease, aspiration pneumonia, peripheral artery disease and osteoporosis.

In elderly hypertensive patients with cerebrovascular disease (chronic phase), it is important to maintain the cerebral blood flow, and blood pressure should be reduced slowly, particularly after cerebral infarction. Ca antagonists, ARB/ACE inhibitors and diuretics (combined with the first two) are used. In the PROGRESS (mean age, $64 \pm 10$ years), a combination of an ACE inhibitor (perindopril) and a diuretic (indapamide) was useful for the prevention of recurrence (secondary prevention). ${ }^{135}$ In the MOSES (mean age, $68 \pm 10$ years), an ARB (eprosartan) was more useful than a Ca antagonist (nitrendipine) for the prevention of recurrence (secondary prevention). ${ }^{320}$

Although there is no specific point regarding the selection of drugs for elderly hypertensive patients with ischemic heart disease or heart failure, $\beta$-blockers should be administered with particular caution toward adverse effects.

In elderly hypertensive patients with nephropathy, blood pressure control as well as dietary therapy is important. Their treatment is basically the same as that for hypertensive patients with chronic kidney disease in general using an ARB/ACE inhibitor as a primary drug. However, the participants of most large-scale clinical studies were aged $\leqslant 70$ years, and there is little evidence for the drug selection or target of blood pressure control, particularly, for patients aged $\geqslant 75$ years. Careful blood pressure control with sufficient attention to changes in renal function, proteinuria and albuminuria is required.

In elderly hypertensive patients with diabetes mellitus, aggressive antihypertensive treatment as well as the management of diabetes is necessary. The choice of antihypertensive drugs and setting of the target blood pressure are the same as those for general treatment for hypertensive patients with diabetes mellitus using an ARB/ACE inhibitor as a primary drug. However, few studies have been conducted in participants including those in late old age, similarly to studies in hypertensive patients with nephropathy, and the partici- 
Table 8-2 Indication of antihypertensive drugs for elderly hypertensive patients with complications

\begin{tabular}{|c|c|c|c|c|}
\hline Complication & Ca antagonists (dihydropyridine) & ARB/ACE inhibitors & Diuretics & $\beta$-Blockers \\
\hline Ischemic heart disease & 0 & 0 & & $\mathrm{Ob}^{\mathrm{b}}$ \\
\hline Chronic kidney disease & $O^{d}$ & $\mathrm{O}^{\mathrm{e}}$ & $O^{d, f}$ & \\
\hline Diabetes mellitus & $O^{d}$ & 0 & $\Delta$ & $\Delta$ \\
\hline Dyslipidemia & O & O & $\Delta$ & $\Delta$ \\
\hline Aspiration pneumoniah & & ACE inhibitors & & $x$ \\
\hline Peripheral artery disease & O & 0 & $\Delta$ & \\
\hline Osteoporosis & & & $O^{i}$ & \\
\hline
\end{tabular}

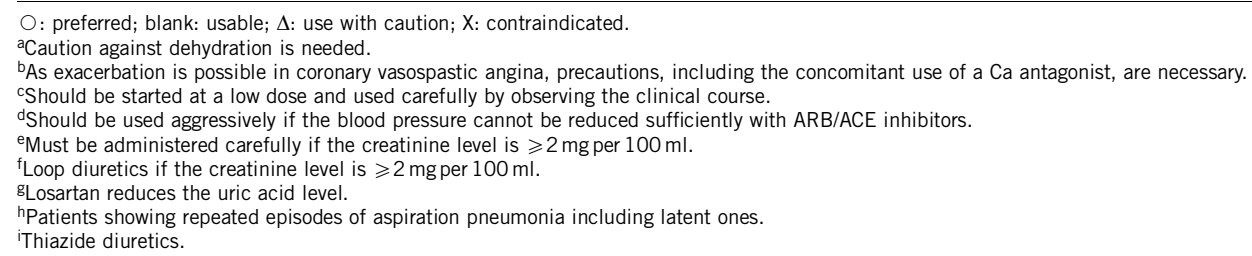

pants in many studies were aged $\leqslant 75$ years. Large-scale clinical studies in which subanalysis involving patients with diabetes was performed include the PROGRESS (761 patients, mean age: 64 years), ${ }^{560}$ Syst-Eur (492 patients, mean age: 70 years) ${ }^{489}$ HOT (1501 patients, mean age: 62 years), ${ }^{139}$ STOP-2 (719 patients, mean age: 76 years), ${ }^{561}$ LIFE (1195 patients, mean age: 67 years), ${ }^{274}$ INSIGHT (1302 patients, mean age: 65 years) ${ }^{562}$ and ALLHAT (13 101 patients, mean age: 67 years). ${ }^{563}$ The treatment was compared with a placebo in the PROGRESS and Syst-Eur, and although reducing the blood pressure has been confirmed to be effective for the prevention of stroke and cardiovascular events, blood pressure could not be reduced to $<130$ / $80 \mathrm{~mm} \mathrm{Hg}$.

The information concerning other complications and drug selection provided in Table 8-2 is not based on evidence such as a decrease in cardiovascular risk, but includes recommendations and cautions based on pharmacological actions and so on. Thiazide diuretics may contribute to the prevention of osteoporosis because of their Ca excretioninhibiting action. As their effects on fracture are being evaluated in the HYVET, their usefulness for the management of osteoporosis is expected to be clarified.

\section{e. Other points requiring attention}

In elderly patients with refractory hypertension, attention not only to secondary hypertension such as endocrine and renal parenchymal hypertension but also to drug-induced hypertension is necessary.
Inquiry about the use of health foods and supplements as well as prescriptions at other hospitals and departments, and about the use of glycyrrhiza and non-steroidal anti-inflammatory drugs is essential.

Another point regarding refractory hypertension relates to medication adherence. Long-acting drugs with a trough/peak ratio of $\geqslant 50 \%$ effectiveness for the control of morning hypertension by 1 or 2 administrations per day are recommended. Declines in medication adherence associated with dementia must also be considered in elderly patients. The possibility of forgetting to take drugs because of impairment of cognitive function should be evaluated even in patients who are capable of communication during the medical examination. Compliance management by the family or caregiver may be necessary. The packing of drugs into one bag is effective not only for maintaining adherence but also for enhancing the effect of medication in elderly patients. $^{214}$

\section{Citation Information}

We recommend that any citations to information in the Guidelines are presented in the following format:

The Japanese Society of Hypertension Guidelines for the Management of Hypertension (JSH 2009). Hypertens Res 2009; 32: 3-107.

Please refer to the title page for the full list of authors. 